\title{
What is new about somatosensory evoked potentials as neurological predictors of comatose survivors after cardiac arrest?
}

\author{
Maenia Scarpino1,2, Giovanni Lanzo ${ }^{1}$, Aldo Amantini ${ }^{1,2}$ \& Antonello Grippo*,1,2 \\ ${ }^{1}$ Servizio di Neurofisiopatologia, IRCCS Fondazione Don Carlo Gnocchi, Firenze, Italy \\ ${ }^{2}$ SODc Neurofisiopatologia, Dipartimento Neuromuscolo-Scheletrico e degli Organi di Senso, AOU Careggi, Firenze, Italy \\ *Author for correspondence: agrippo@unifi.it
}

First draft submitted: 20 January 2020; Accepted for publication: 7 February 2020; Published online: 24 April 2020

Keywords: brain computed tomography $\bullet$ cardiac arrest $\bullet$ cerebral performance categories $\bullet$ electroencephalogram - hypoxic ischemic encephalopathy $\bullet$ multimodal approach $\bullet$ neurological outcome $\bullet$ pupillary reflex $\bullet$ somatosensory evoked potentials

\section{Long-term neurological prediction of hypoxic ischemic encephalopathy}

Hypoxic ischemic encephalopathy (HIE) is a frequent and severe neurological complication after cardiac arrest (CA). HIE is often related to a poor neurological outcome (death or persistent vegetative state), due to severe and large hypoxic ischemic brain damage. However, sometimes it may be related to a better neurological outcome (recovery of consciousness with severe or minor disability), because it has been caused by a minor brain injury. Neurological outcome prediction of comatose HIE patients is still challenging for physicians, mainly in the early stages after CA and to date it still represents an important goal. In fact, an early but accurate and reliable neurological prognosis, on the one hand, allows optimization of the diagnostic-therapeutic pathway of CA patients among ICUs, such as for the patients who undergo invasive treatments. While, on the other hand, it may improve communication with the patient's family. Moreover, a reliable neurological prognosis may allow physicians to identify the best postacute care, such as an intensive rehabilitation unit or long-term care, in accordance with the real expectations regarding the clinical recovery of the patients.

\section{The multimodal approach}

Given the great relevance of this topic, several studies and guidelines for postresuscitation care of CA patients have been proposed in recent years in the literature. In particular, the European Resuscitation Council and European Society of Intensive Care Medicine co-issued the actual guidelines for postresuscitation care [1], suggesting a multimodal approach for a reliable neurological prognosis of HIE. To be more specific, these guidelines, according to the evidence of literature relating to those years, identified a clinical assessment, based on pupillary reflex evaluation and short-latency somatosensory evoked potentials (SEPs) as robust predictors, whereas electroencephalogram (EEG), brain computed tomography, serum biomarkers and early myoclonus were identified as less robust predictors.

\section{SEPs as robust neurological predictors of comatose survivors after CA}

In recent years, SEPs have been evaluated as neurological outcome predictors of CA patients in many studies.

However, in most of these papers [2,3], the clinician adopted withdrawal of life-sustaining treatment protocols, using SEPs as index test, then creating a self-fulfilling prophecy bias.

Another limitation was related to the definition of poor neurological outcome, because some studies identified death (cerebral performance categories [CPC] 5) and a persistent vegetative state (PVS; CPC 4) as ominous outcomes [4,5], whereas other work also defined severe neurological disability (CPC 3) as a poor outcome [2,3].

Robinson et al. [6] were the first authors who suggested that the bilaterally absent (AA) cortical SEP pattern was related to nonrecovery of the conscious state.

Some years later, Wijdicks et al. [7] confirmed these results and suggested that comatose patients with a SEP pattern AA at $72 \mathrm{~h}$ after CA demonstrated a long-term poor neurological outcome. Moreover, according to Wijdicks

Future Medicine 
et al. and the American Academy of Neurology guidelines, some clinical parameters observed at $72 \mathrm{~h}$ after CA, such as bilaterally absent pupillary and corneal reflexes, a motor Glasgow Coma Scale score of $\leq 2$ and the presence of a myoclonus, had the same poor prognostic meaning as the SEP pattern AA. However, during the same period, the increase in the use of the Targeted Temperature Management (TTM) protocol in the clinical practice of CA patients had affected the prognostic value of the clinical features included in the American Academy of Neurology guidelines, when observed at $72 \mathrm{~h}$ after CA. In particular, some studies [8,9], demonstrated that only the SEP pattern AA kept its poor prognostic power unchanged during TTM, in contrast to clinical parameters. These results were also confirmed in a later study by Grippo et al., [4] in which the authors reported that the SEP pattern AA performed during TTM had the same poor prognostic power as that obtained after the end of the TTM protocol.

\section{Limitations of SEPs}

According to the majority of the literature, SEPs have a false-positive rate of zero percent (CI: 0-3) [1] for an outcome no better than PVS. Repeated testing should always be considered when cortical responses N20 are present but with severe reduced amplitude at an early stage (12-24 h) after CA, as they may later disappear. However, despite this homogeneity of thought among the authors, in a multicenter cohort representing clinical practice [9], the interobserver agreement of SEP interpretation in patients with hypoxic ischemic coma was only moderate. The noise level strongly influenced interobserver disagreement. Finally, in another study [10], the authors demonstrated that neurologic recovery was possible in a small number of patients (one out of 36 patients) treated with induced hypothermia after CA and with bilaterally absent N20 responses. However, the authors did not report possible variables such as technical factors or neuroimaging data in their study.

\section{Updating on the use of SEPs as neurological predictors of comatose patients after CA}

Although, the SEP index shows high specificity, they are able to identify only about $50 \%$ of patients with poor neurological outcome [11,12]. Moreover, the bilateral presence of N20 responses does not ensure a good outcome [7]. For this reason, authors have suggested in most recent studies $[1,13]$, that the use of a multimodal approach based on the evaluation of clinical, serum biomarkers and instrumental predictors should be used in clinical practice, in order to improve both the sensitivity and reliability of a poor outcome prediction, in addition to the identification of a subset of CA patients demonstrating a good outcome. According to the results of these studies [5,14-17], authors observed that a SEP pattern absent-pathological (AP), where pathological and amplitude of the cortical wave $<1.2 \mu \mathrm{V}$ was concerned, had the same poor prognostic meaning as the SEP pattern AA. The strength of these works was represented by the absence of withdrawal of life-sustaining treatment in the subset of CA patients analyzed. This allowed the risk of self-fulfilling prophecy bias to be minimized and the natural neurological evolution of these patients to be observed.

In the same period, the marked bilateral amplitude reduction of SEP cortical responses (PP) was associated with a poor neurological outcome, similar to the SEP pattern AA [18,19]. In particular, in a study by Carrai et al., [18], the authors observed that amplitude reduction values of $<0.65 \mu \mathrm{V}$ for both cortical SEPs were associated with a poor long-term neurological outcome.

The identification of other SEP patterns with the same poor prognostic meaning as the SEP pattern AA represents an important goal, because it improved the correct classification of CA patients, thereby increasing the identification of subjects which demonstrate a poor outcome.

In fact, both the identification of more SEP patterns related to a poor outcome and the use of a multimodal approach based on different clinical and instrumental predictors, on the one hand, allowed the correct identification of a greater number of patients with a poor outcome. While, on the other hand, they allowed the identification of patients demonstrating a good outcome (although limited to the finding of specific EEG patterns at an early stage $[\leq 12 \mathrm{~h}]$ after CA) $[17,20]$.

An increased correct prediction of neurological outcome, based on all the parameters reported above, is important for reducing the prognostic uncertainty that would result from an approach based on the use of only a single clinical or instrumental predictor or on the use of only SEP pattern AA, as an index of poor neurological outcome.

\section{SEPs as predictors of evolution toward brain death in patients with HIE}

Organ transplantation is an increasingly widespread procedure, as it is the only available treatment for certain end-stage organ diseases. However, due to the disproportion between the number of patients on the waiting list for transplantable organs and the availability of organs to donate, the optimization of old strategies may be necessary, 
along with the implementation of new ones aimed at detecting all possible organ donors. For this reason, early identification of possible organ donors among patients affected by brain injuries, such as HIE, that less frequently demonstrate an evolution toward brain death (BD), would be an important goal.

In this perspective, SEPs have been evaluated as early predictors of BD in patients with HIE [21]. These authors demonstrated that the SEP patterns AA and AP were related to an early evolution toward $\mathrm{BD}$, with high specificity but low sensitivity, since the majority of patients with these SEP patterns remained in a PVS.

However, in another work by the same group [22], the authors, in addition to having confirmed that the risk of evolution toward BD was related only to the presence of an AA or AP SEP pattern, demonstrated that the presence of a density ratio value of $<1.07$ between gray matter and white matter at the basal ganglia level, evaluated by brain computed tomography, was highly suggestive of an early evolution toward BD.

\section{Conclusion}

Prognosticating the neurological outcome of resuscitated patients after CA is still challenging and requires a multimodal approach. Among the neurophysiological tests suggested by current guidelines [1], SEPs are considered a robust predictor, demonstrating strong and time-independent results, as the poor predictive meaning of an AA SEP pattern is the same at any time after CA. Moreover, in the earlier stages after CA $(\leq 12 \mathrm{~h})$, SEPs provide information complementary to that of an EEG. Thus, the use of both these neurophysiological tests enables correct classification of a greater number of patients, as an AA SEP pattern enables the identification of CA comatose survivor patients with a poor outcome, while a continuous, nearly continuous or low-voltage EEG pattern enables the identification of CA patients with a good outcome [17].

With regards to the poor prognostic meaning of other SEP patterns (such as AP and PP), several studies are needed to confirm the preliminary results obtained in previous works. In particular, where the PP pattern is concerned, further multicenter and prospective studies are necessary to strengthen its poor prognostic meaning, in order to be used like the SEP pattern AA in routine clinical practice.

\section{Financial \& competing interests disclosure}

The authors have no relevant affiliations or financial involvement with any organization or entity with a financial interest in or financial conflict with the subject matter or materials discussed in the manuscript. This includes employment, consultancies, honoraria, stock ownership or options, expert testimony, grants or patents received or pending or royalties.

No writing assistance was utilized in the production of this manuscript.

\section{Open access}

This work is licensed under the Attribution-NonCommercial-NoDerivatives 4.0 Unported License. To view a copy of this license, visit http://creativecommons.org/licenses/by-nc-nd/4.0/

\section{References}

Papers of special note have been highlighted as: $\bullet$ of interest; $\bullet \bullet$ of considerable interest

1. Nolan JP, Soar J, Cariou A et al. European Resuscitation Council and European Society of Intensive Care Medicine Guidelines for post-resuscitation care 2015: section 5 of the European Resuscitation Council Guidelines for resuscitation 2015. Resuscitation 95 , 202-222 (2015).

-. Most recent consensus regarding prognostication of patients survived after cardiac arrest.

2. Tsetsou S, Novy J, Pfeiffer C, Oddo M, Rossetti AO. Multimodal outcome prognostication after cardiac arrest and targeted temperature management: analysis at 36 . Neurocrit. Care 28, 104-109 (2018).

3. Glimmerveen AB, Ruijter BJ, Keijzer HM, Tjepkema-Cloostermans MC, van Putten MJAM, Hofmeijer J. Association between somatosensory evoked potentials and EEG in comatose patients after cardiac arrest. Clin. Neurophysiol. 130, 2026-2031 (2019).

4. Grippo A, Carrai R, Fossi S et al. Absent SEP during therapeutic hypothermia did not reappear after re-warming in comatose patients following cardiac arrest. Minerva Anestesiol. 79, 360-369 (2013).

5. Scarpino M, Lanzo G, Lolli F et al. Neurophysiological and neuroradiological multimodal approach for early poor outcome prediction after cardiac arrest. Resuscitation 129, 114-120 (2018).

6. Robinson LR, Micklesen PJ, Tirschwell DL, Lew HL. Predictive value of somatosensory evoked potentials for awakening from coma. Crit. Care Med. 31, 960-967 (2003).

- First systematic review concerning the use of somatosensory evoked potentials for prognostication in comatose patients of several etiologies, including cardiac arrest. 
7. Wijdicks EF, Hijdra A, Young GB et al. Practice parameter: prediction of outcome in comatose survivors after cardiopulmonary resuscitation (an evidence-based review): report of the Quality Standards Subcommittee of the American Academy of Neurology. Neurology 67, 203 (2006).

- First guideline regarding the prognostication of patients surviving cardiac arrest.

8. Rossetti AO, Oddo M, Logroscino G, Kaplan PW. Prognostication after cardiac arrest and hypothermia: a prospective study. Ann. Neurol. 67, 301 (2010).

9. Bouwes A, Binnekade JM, Kuiper MA et al. Prognosis of coma after therapeutic hypothermia: a prospective cohort study. Ann. Neurol. 71, 206 (2012).

10. Leithner C, Ploner CJ, Hasper D, Storm C. Does hypothermia influence the predictive value of bilateral absent N20 after cardiac arrest? Neurology 74, 965 (2010).

11. Sandroni C, Cavallaro F, Callaway CW et al. Predictors of poor neurological outcome in adult comatose survivors of cardiac arrest: a systematic review and meta-analysis. Part 2: patients treated with therapeutic hypothermia. Resuscitation 84, 1324-1338 (2013).

-• Most recent consensus regarding prognostication of patients survived after cardiac arrest.

12. Scarpino M, Grippo A, Lanzo G et al. The burden of clinical neurophysiology for the neurological prognosis of coma. Future Neurol. 13, 127-129 (2018).

- A short, updated summary regarding the use of electroencephalogram and somatosensory evoked potentials for prognostication of comatose patients affected by acquired brain injury.

13. Taccone FS, Baar I, De Deyne C et al. Neuroprognostication after adult cardiac arrest treated with targeted temperature management: task force for Belgian recommendations. Acta Neurol. Belg. 117, 3-15 (2017).

14. Scarpino M, Lolli F, Lanzo G et al. Neurophysiology and neuroimaging accurately predict poor neurological outcome within 24 hours after cardiac arrest: the ProNeCA prospective multicentre prognostication study. Resuscitation 143, 115-123 (2019).

15. Scarpino M, Lanzo G, Lolli F et al. Data on multimodal approach for early poor outcome (cerebral performance categories 3-5) prediction after cardiac arrest. Data Brief 19, 704-711 (2018).

16. Scarpino M, Lolli F, Lanzo G et al. Neurophysiological and neuroradiological test for early poor outcome (cerebral performance categories 3-5) prediction after cardiac arrest: prospective multicentre prognostication data. Data Brief 27, 104755 (2019).

17. Scarpino M, Carrai R, Lolli F et al. Neurophysiology for predicting good and poor neurological outcome at 12 and $72 \mathrm{~h}$ after cardiac arrest: the ProNeCA multicentre prospective study. Resuscitation 147 95-103 ( 2019).

18. Carrai R, Scarpino M, Lolli F et al. Early-SEPs' amplitude reduction is reliable for poor-outcome prediction after cardiac arrest? Acta Neurol. Scand. 139, 158-165 (2019).

19. Endisch C, Storm C, Ploner CJ, Leithner C. Amplitudes of SSEP and outcome in cardiac arrest survivors: a prospective cohort study. Neurology 85, 1752-1760 (2015).

20. Carrai R, Grippo A, Scarpino M et al. Time-dependent and independent neurophysiological indicators of prognosis in post-anoxic coma subjects treated by therapeutic hypothermia. Minerva Anestesiol. 82, 940-949 (2016).

21. Scarpino M, Lanzo G, Carrai R et al. Predictive patterns of sensory evoked potentials in comatose brain injured patients evolving to brain death. Neurophysiol. Clin. 47, 19-29 (2017).

22. Scarpino M, Lanzo G, Lolli F et al. Is brain computed tomography combined with somatosensory evoked potentials useful in the prediction of brain death after cardiac arrest? Neurophysiol. Clin. 47, 327-335 (2017). 